\title{
Ultrasensitive detection of endocrine disruptors via superfine plasmonic spectral combs
}

\author{
Lanhua Liu', Xuejun Zhang $\mathbb{D}^{2}$, Qian Zhu', Kaiwei Li', Yun Lu', Xiaohong Zhou' ${ }^{1 凶}$ and Tuan Guo $\mathbb{B}^{2 凶}$
}

\begin{abstract}
The apparent increase in hormone-induced cancers and disorders of the reproductive tract has led to a growing demand for new technologies capable of detecting endocrine disruptors. However, a long-lasting challenge unaddressed is how to achieve ultrahigh sensitive, continuous, and in situ measurement with a portable device for infield and remote environmental monitoring. Here we demonstrate a simple-to-implement plasmonic optical fiber biosensing platform to achieve an improved light-matter interaction and advanced surface chemistry for ultrasensitive detection of endocrine disruptors. Our platform is based on a gold-coated highly tilted fiber Bragg grating that excites high-density narrow cladding mode spectral combs that overlap with the broad absorption of the surface plasmon for high accuracy interrogation, hence enabling the ultrasensitive monitoring of refractive index changes at the fiber surface. Through the use of estrogen receptors as the model, we design an estradiol-streptavidin conjugate with the assistance of molecular dynamics, converting the specific recognition of environmental estrogens (EEs) by estrogen receptor into surface-based affinity bioassay for protein. The ultrasensitive platform with conjugate-induced amplification biosensing approach enables the subsequent detection for EEs down to $1.5 \times 10^{-3} \mathrm{ng} \mathrm{ml}^{-1}$ estradiol equivalent concentration level, which is one order lower than the defined maximal $E_{2}$ level in drinking water set by the Japanese government. The capability to detect EEs down to nanogram per liter level is the lowest limit of detection for any estrogen receptor-based detection reported thus far. Its compact size, flexible shape, and remote operation capability open the way for detecting other endocrine disruptors with ultrahigh sensitivity and in various hard-toreach spaces, thereby having the potential to revolutionize environment and health monitoring.
\end{abstract}

\section{Introduction}

Environmental estrogens (EEs), as typical endocrine disruptors, are structurally diverse compounds that can interact with nuclear estrogen receptors (nERs) and pose significant risks to human and ecological health ${ }^{1}$. A large number EEs are improperly released into the environment due to human activities (e.g., industrial, agricultural, urban), especially the huge estrogenic drug consumption world widely ${ }^{2,3}$. As a consequence, EE contamination has been listed as one of the global environmental issues to be addressed through international collaboration by the

\footnotetext{
Correspondence: Xiaohong Zhou (xhzhou@mail.tsinghua.edu.cn) or Tuan Guo (tuanguo@jnu.edu.cn)

'State Key Joint Laboratory of ESPC, School of Environment, Tsinghua University, Beijing 100084, China

${ }^{2}$ Institute of Photonics Technology, Jinan University, Guangzhou 510632, China

These authors contributed equally: Lanhua Liu, Xuejun Zhang, Qian Zhu
}

United Nations. A variety of technologies have been applied in the field of estrogen detection (Fig. S1). Because of the structural diversity of EEs, it is difficult to detect all broad ranges of EEs by using many conventional structure-specific instrumental analytical methods, such as high-performance liquid chromatography-mass spectrometry and gas chromatography-mass spectrometry. Notably, these methods are powerless to the unknown ones. Therefore, developing the advanced and powerful detection technologies to characterize as many EEs as possible in the environment is still challenging, however, highly demanded.

By mimicking the function of the natural estrogens or disturbing the signal transduction pathways for estrogens, EEs could induce abnormality in the growth, development, and maintenance of the reproductive system $^{4,5}$. nERs are members of the steroid receptor family

\section{(c) The Author(s) 2021}

(c) (i) Open Access This article is licensed under a Creative Commons Attribution 4.0 International License, which permits use, sharing, adaptation, distribution and reproduction cc) in any medium or format, as long as you give appropriate credit to the original author(s) and the source, provide a link to the Creative Commons license, and indicate if changes were made. The images or other third party material in this article are included in the article's Creative Commons license, unless indicated otherwise in a credit line to the material. If material is not included in the article's Creative Commons license and your intended use is not permitted by statutory regulation or exceeds the permitted use, you will need to obtain permission directly from the copyright holder. To view a copy of this license, visit http://creativecommons.org/licenses/by/4.0/. 
of nuclear receptors and mediate most estrogen responses in the body. The binding interaction between EEs and nERs is identified as one of the molecular initiative events of estrogen signal transduction pathways, leading to the subsequent downstream events, such as nER-mediated cell proliferation ${ }^{6-8}$. Based on the specific interactions between EEs and nERs, many cellbased in vitro assays are established, e.g., ER-CALUX®, the MELN, and the Yeast Estrogen Screen (YES) assays, to provide effective and robust detection of broad ranges of $\mathrm{EEs}^{9}$. However, the majority of these bioassays require days-long operations, specialized experts, or sophisticated equipment. Notably, the cell-based bioassays are confronted with the problem of cytotoxic substances, which may be present in environmental samples and could lead to erroneous results unless proper controls are included ${ }^{10}$.

As an alternative to cell-based bioassays, cell-free approaches offer in vitro protein expression for analytic purposes. By using nER protein as a recognition element, the competitive binding assays have also been established by using radioactive estradiol (17 $\beta$-Estradiol $\left.\left(\mathrm{E}_{2}\right)\right)$, such as $[3 \mathrm{H}] \mathrm{E}_{2}{ }^{11,12}$ or $[125 \mathrm{I}] \mathrm{E}_{2}{ }^{13}$, as the signal reporter. It is worth mentioning that such competitive nER binding in vitro experiment has been listed as the first scheme of endocrine-disruptor screening program by the US Environmental Protection Agency. However, the reported nER competitive binding assays mostly rely on the homogeneous system, and the real-time, continuous, and intelligent detection of EEs has not yet been fully developed. With the flourishing development of materials science and synthetic biology, biosensor technology is expected to break through the above issues.

To date, numerous nER-based biosensors have been developed by using different signal transductions modes, such as fluorescence ${ }^{14}$, surface plasmon resonance $(\mathrm{SPR})^{15-17}$, piezoelectric ${ }^{18}$, and electrochemistry ${ }^{19,20}$. These cell-free biosensors are cost-effective and robust to the cytotoxicity of environmental samples. They are useful complements to existing laboratory methods, facilitating quick and affordable identification of all EEs that act through the nERs in remote, non-laboratory settings ${ }^{1,14}$. However, most of them are still blamed with inferior sensitivities largely due to the binding affinity limitation of nER protein, especially used for the analysis of EEs commonly existing at trace concentrations in the environment. As an example, the defined maximal $E_{2}$ level in drinking water is set to be $0.08 \mathrm{ng} \mathrm{ml}^{-1}$ by the Japanese government ${ }^{21}$. Hence, developing an advanced and sensitive nER-based biosensing platform is highly demanded, however, still challenging.

Recently, a new promising class of label-free optical biosensors, i.e., fiber-optic SPR biosensor, has emerged due to their desirable features, such as the suitability to miniaturization, remote sensing, and ease of use compared with the well-established bulky prism configurations (i.e., Kretschmann-Raether prism) ${ }^{22-24}$. These features enable it possible to be inserted into various hard-to-reach environments for in situ detection either as a hand-held probe or as a set of remotely operated devices fixed at various locations along a fiber-optic cable. The most common fiber-optic SPR sensors are made from cladding-modified optical fibers (unclad, side-polished, tapered, and U-shaped) covered with a nanometric layer of gold or silver ${ }^{25}$. More recently, the fiber grating technology based on tilted fiber Bragg grating (TFBG) has been developed for the construction of a highly efficient SPR sensor with several unique advantages ${ }^{26,27}$. Such sensor provides an additional resonant mechanism of high-density narrow cladding mode spectral combs at the near-infrared wavelength (with a spectral width of the resonance between 0.01 and $0.1 \mathrm{~nm}$ ) that overlaps with the broad absorption of the surface plasmon for high accuracy interrogation, which rises the resolution of refractive index (RI) from $10^{-6}$ to $10^{-8}$ RIU (refractive index unit) and offers a linear RI response in both air and liquids ${ }^{28,29}$. Second, the mechanical resistance of the TFBG sensor is minimally impacted and its mass production with good reproducibility can be easily achieved by well-established phase masks for grating inscription. Last but not least, the temperature cross-sensitivity can be suppressed by calibrating the light remaining in the core of the TFBG fiber, i.e., the Bragg resonance, which is inherently insensitive to the external RI changes unless acting as a thermometer. Even with the excellent features mentioned above, the research on TFBG-based fiber-optic SPR technology used for ultrasensitive detection of broad ranges of EEs via ER has never been reported. The robust biosensor design with advanced surface chemistry of TFBG-based fiber-optic and rational biosensing strategy for EEs is still the challenge to be addressed.

To address the above research gap, we developed a gold film-coated TFBG-based SPR biosensor for the ultrasensitive screening of EEs. Through the covalent surface modification of plasmonic fiber optic, the specific interaction of nERs and EEs was converted into measurable signals to realize the facile detection of EEs in environmental samples. Benefiting from the ultrahigh sensitivity of gold film-coated TFBG-based SPR biosensing platform and the broad recognition capability of $n E R s$, the water samples containing EEs in the nanogram per liter estradiol equivalent concentration (EEQ) range were determined by the biosensor with satisfactory recovery rates. Such sensor offers an ideal solution to meet the strategic layout of continuous assessment of global environmental endocrine disruptors. 


\section{Results}

\section{Characterization of plasmonic optical fiber sensor}

To achieve specificity of detection, functionalization of plasmonic optical fiber to effectively discriminate the $\mathrm{E}_{2}$-streptavidin (STV) was indispensable. The wellestablished strategy to functionalize functional groups/ molecules onto gold film-coated SPR surface is via Au$\mathrm{S}$ bond by using thiol-end organic compounds to form self-assembled monolayers (SAMs) spontaneously ${ }^{30}$. Desthiobiotin-polyethylene glycol-thiol (DTB-PEG$\mathrm{SH}$ ) was used to form SAMs on gold surface, which offered the exposed DTB to bind the released $E_{2}-S T V$ conjugate efficiently, while the bovine serum albumin (BSA) solution as a blocking agent was incubated to block the nonspecific binding sites and enhance the specific STV-DTB interaction, and hence increase the signal/noise ratio. Moreover, the PEG linker between -SH and DTB offered better water solubility and reduced the steric hindrance to enhance the reactivity of DTB.

The functionalization of the Au-coated TFBG fiber followed the procedures below, as shown in Fig. 1a. First, the probe was immersed in the deionized (DI) water for $66 \mathrm{~min}$ to stabilize this sensor, which can eliminate environmental interference to verify the stability of sensing system. Second, bare gold was functionalized with DTB-PEG-SH crosslinker and then coated with the BSA blocker for 67 and $33 \mathrm{~min}$, respectively. Notably, the DI water was used to rinse the probe for $10 \mathrm{~min}$ to remove the residuals at the end of both procedures of DTB-PEG-SH and BSA modification. Figure $1 \mathrm{~b}$ shows the variable spectra recorded every $20 \mathrm{~s}$ under the above three different conditions: pure gold film-coated TFBG in water (black curve); gold film-coated TFBG modified with DTB-PEG-SH (red curve) and then blocked with BSA solution (blue curve). Three modes with the highest sensitivity were selected, respectively, and marked as (1), (2), and (3). Based on the above results, the intensity variations of gold film-coated TFBG as a function of time during the water stability and the DTB-PEG-SH and BSA adsorption on gold surface, respectively, are demonstrated at each selected mode and compared in Fig. 1c. Its inset shows the atomic force microscopic (AFM) images of the morphology of bare and DTB-PEG-SH-modified plasmonic optical fiber. Extensive analysis of the AFM topography cross-sections (Fig. S2) showed that the surface thickness of the chemically modified fiber was slightly increased than that of the bare fiber considering the small size of the DTB-PEG-SH. The response curve versus time also clearly demonstrated that the intensity was stable at the selected highly sensitive mode (1) in water while changed when the TFBG fiber was modified by DTB-PEG-SH and BSA.

\section{Design of $\mathrm{E}_{2}-\mathrm{STV}$ conjugate}

The length and structure of the linker between the $E_{2}$ and STV are critical factors affecting the binding performance of the $\mathrm{E}_{2}$-STV conjugate and $\mathrm{hER} \alpha$ ligand-binding domain (LBD). Although the flexible and rigid linkers in the design of estradiol derivatives were considered, the ligands with rigid linkers showed unavoidable atomic position conflict with $\mathrm{hER} \alpha \mathrm{LBD}$, indicating that the rigid structure was not suitable for the linker design for $\mathrm{E}_{2}-\mathrm{STV}$ conjugates (Fig. S3). Among estradiol derivatives with flexible linkers, estradiol derivative 4 was docked to hERLBD as shown in Fig. S4, and the $-\mathrm{COOH}$ terminal was blocked in the hER $\alpha \mathrm{LBD}$, showing that the linker was too short to meet the requirement of connecting STV, so that no further simulation will be performed in the follow-up.

Figure 2 summarizes the molecular dynamics (MD) simulation results of carbonylated estradiol derivatives, named $8,11,16$, and 20 with different numbers of flexible carbon skeleton as shown in Table 1 . The root-meansquare deviation (RMSD) is an accepted index to measure the conformational changes of the MD simulation systems. Figure 2a displays the time evolution of RMSDs for $\mathrm{hER} \alpha \mathrm{LBD}$ in the simulations with four carbonylated estradiol derivatives, respectively. As expected, a plateau was reached with the RMSDs of all complexes $<1.0 \mathrm{~nm}$ within $10 \mathrm{~ns}$. The representative snapshots of complexes at the end of simulations are shown as in Fig. 2a, and the complexes of estradiol derivatives (shown in green) and $h E R \alpha$ LBD were compared with the original $E_{2}-h E R \alpha$ LBD crystal structure, where $E_{2}$ is shown in magenta. The comparison confirmed that the estradiol derivatives maintained the binding conformation with hER $\alpha$ LBD in the MD simulation with the tail of the linking arm fully exposed. It also indicated that adding a flexible linker with appropriate length was an effective way not only to retain the capability being recognized by hER $\alpha$ LBD but also to avoid the possible steric hindrance caused by STV. The root-mean-square fluctuation (RMSF) is a measure of the atomic position deviation in a given length of time defined as the atomic position deviation during MD simulations ${ }^{31}$. The RMSF results further indicated that H12 (residues 531-549) was the most fluctuating part of hER $\alpha$ LBD, while $\mathrm{H} 1-\mathrm{H} 11$ and the ligand-binding pocket usually remained unaffected (Fig. S5). It could be another explanation for why the addition of linkers in the estradiol derivatives had a slight impact on the binding pocket of $h E R \alpha$ LBD for estrogen identification.

Binding energy in the simulations was calculated to predict the binding affinity of hER $\alpha$ LBD and estradiol derivatives. As shown in Fig. 2b, the complex of estradiol derivative 8 and hER $\alpha$ LBD exhibited the lowest average binding energy during the MD simulations, while the simulated binding energies of other derivatives-hER $\alpha$ LBD complex structures were much higher. The results of 


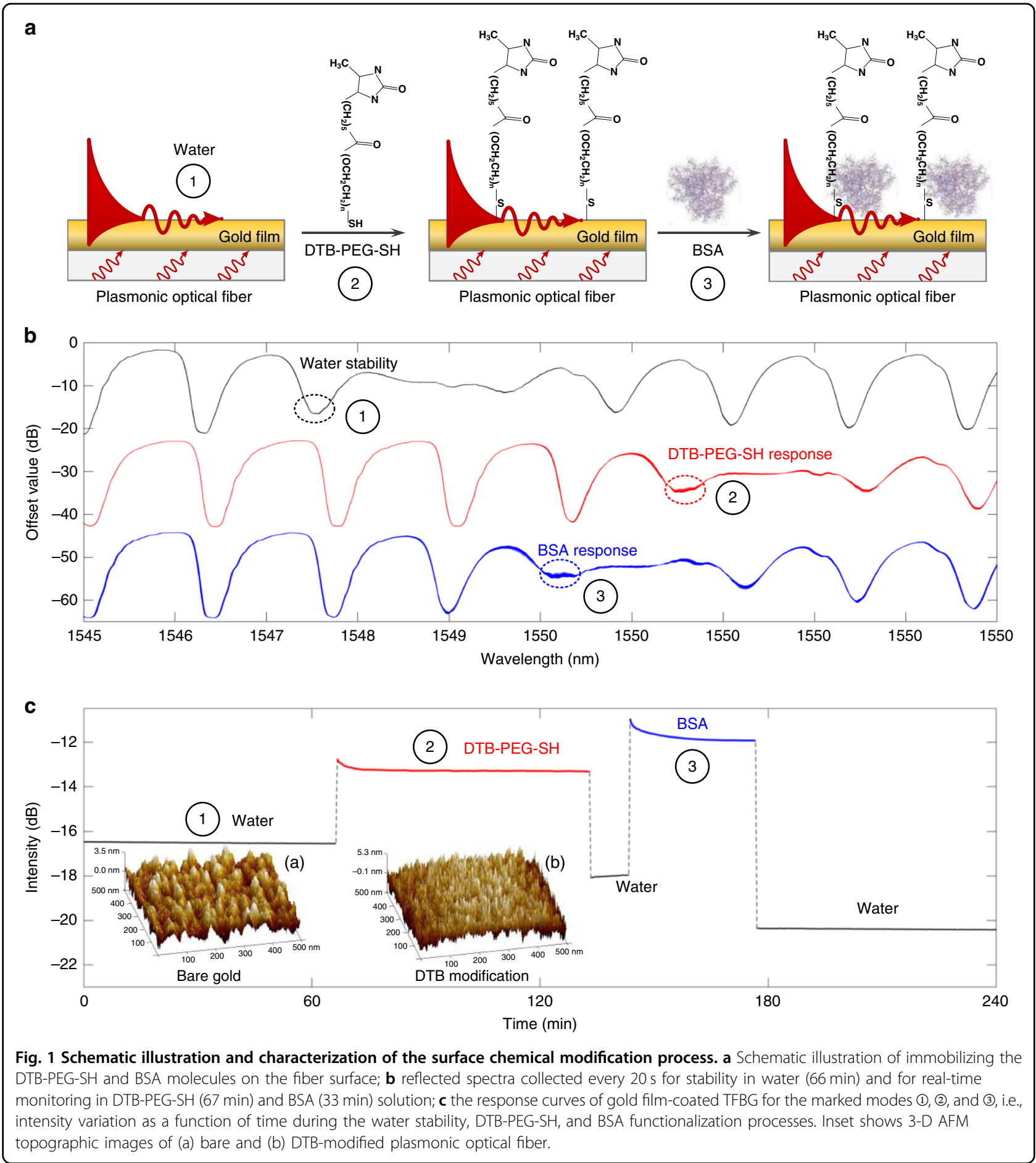

Hbond formation further confirmed that estradiol derivative 8 was able to form more hydrogen bonds to hER $\alpha$ LBD compared with other derivatives, including 11, 16, and 20 (Fig. 2c), therefore resulting in the higher binding affinity of the complex of estradiol derivative 8 and $h E R \alpha$ LBD. Based on the above MD simulation results, estradiol derivative 8 with the most stable binding capability to $h E R \alpha$ LBD was chosen for the synthesis of the $E_{2}-S T V$ conjugate.

\section{Optimization of plasmonic sensing characteristics}

To achieve the best sensing performance of plasmonic optical fiber, the most sensitive plasmonic mode located in the SPR envelope to monitor the interactions of 

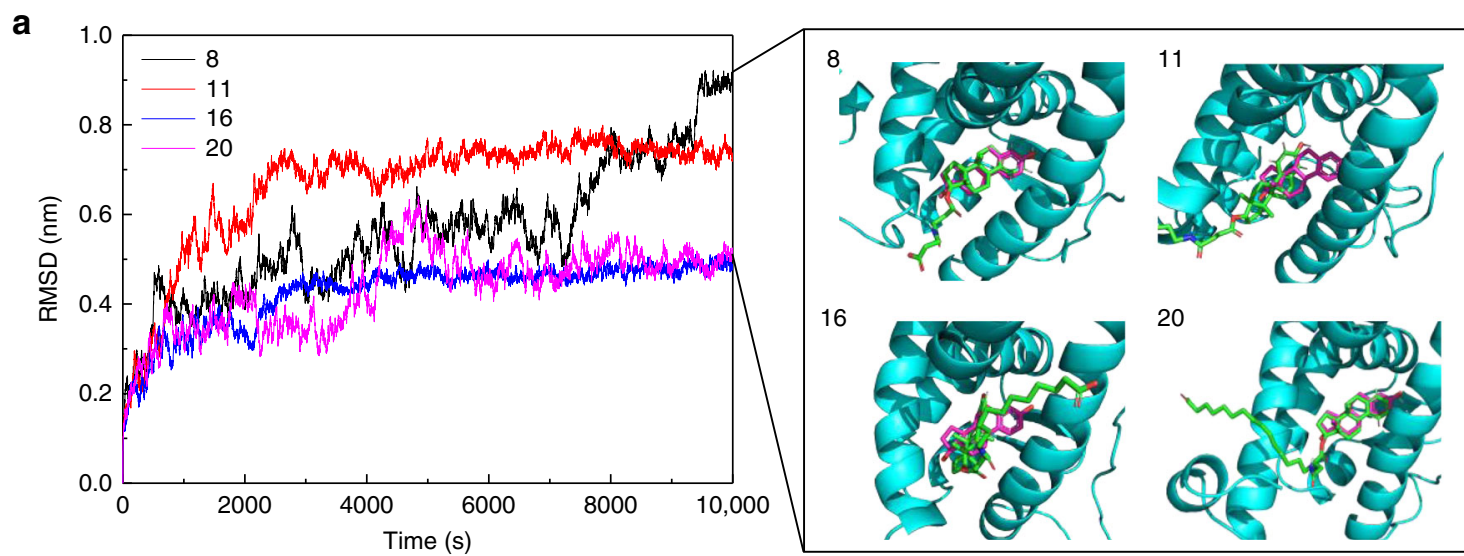

16

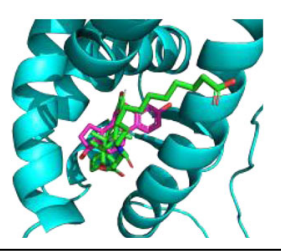

20
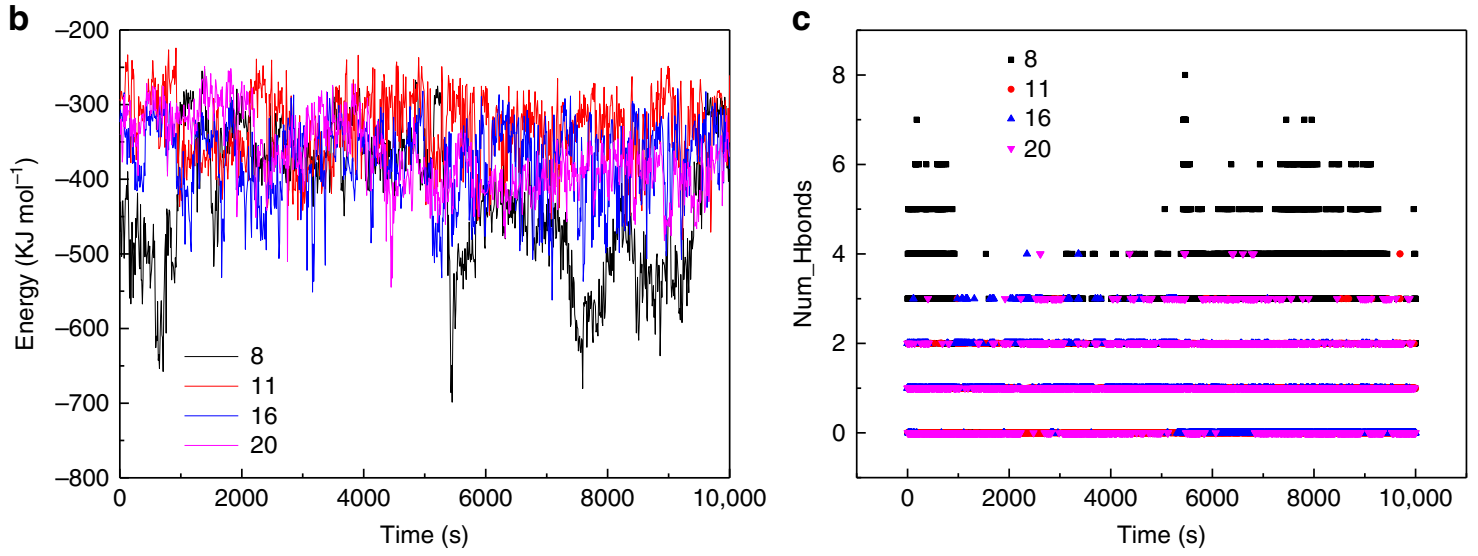

Fig. 2 Design of $E_{2}-S T V$ conjugate based on the MD simulations. a Time evolution of RMSD for the backbone atoms of hERa LBD and estradiol derivatives $8,11,16$, and 20 with different flexible carbon skeleton as shown in Table 1 (left) and the snapshot of the estradiol derivative-hERa LBD complex conformation at the end of MD simulation (right). In the right figure, $\mathrm{E}_{2}$ in magenta is used as a position reference for conformations; $\mathbf{b}$ total non-bond energy of estradiol derivative and hERa LBD complexes; c Hbond number of estradiol derivatives and hERa complexes.

molecules was investigated and validated both experimentally and in silico, the mechanism of which is shown in Fig. S6. Figure 3a shows the cladding mode change of plasmonic optical fiber when modified with the DTBPEG-SH on the gold film in $1 \mathrm{~h}$. As the SPR envelope has a red shift associated with increased RIs, i.e., more molecules attached, the amplitudes of the plasmonic cladding modes (marked as 1, 2, 3, 4) decreased while the other plasmonic modes (marked as 5, 6, 7, 8) increased, and all modes exhibited different relative intensity sensitivities. Among them, the two most sensitive modes 4 and 5 were identified and their zoomed spectral changes are shown in the inset. Figure $3 \mathrm{~b}$ shows the relative intensity changes of the modes $1-8$ during the whole reaction process. The mode 4 closely located at left shoulder of the SPR envelope exhibited the maximum relative intensity response for the DTB-PEG$\mathrm{SH}$ adsorption. Figure $3 \mathrm{c}$ shows the total intensity changes of modes $1-8$ after $1 \mathrm{~h}$ reaction, which exhibited a quantification curve similar to the profile of the SPR envelope.
For better comparison with the experimental data, the simulated results corresponding to the above experimental conditions were conducted. The simulation of the transmission spectrum of the fabricated gold film-coated TFBG was carried out by first solving for the modes of the fiber structure (inclusive of core, cladding, metal layers, high refractive index molecular, and water) with a complex vectorial finite-difference algorithm and then using the coupled mode theory as described previously ${ }^{32}$ for the transmission of TFBG with the aid of MATLAB. The parameters used in the simulation were as follows: a core radius of $4.1 \mu \mathrm{m}$ with a RI of 1.4545 , a cladding radius of $62.5 \mu \mathrm{m}$ with a RI of 1.4467 , a gold coating with a thickness of $50 \mathrm{~nm}$ and complex RI of $0.58-\mathrm{i} \times 11$, and a RI index of 1.3154 for water. The effective RI of surface molecular over gold coating is 1.48 (Fig. S7) ${ }^{33}$. Figure 3d shows simulated transmission spectra of the fabricated gold film-coated TFBG in DI water. It is worth mentioning that there was a sharp decrease in the amplitude of cladding mode resonances in the vicinity of $1550 \mathrm{~nm}$, where those cladding modes had transferred energy to a 
Table 1 Performance comparison of this work with other reported nER-based biosensors for EE detection.

\begin{tabular}{|c|c|c|c|}
\hline Transducer used & $\begin{array}{l}\text { Quantitative range } \\
\left(\mathrm{ng} \mathrm{ml}^{-1}\right)\end{array}$ & $\begin{array}{l}\text { LOD (ng } \\
\mathrm{ml}^{-1} \text { ) }\end{array}$ & Ref. \\
\hline Electrochemical & / & / & 6 \\
\hline Fluorescent & / & / & 13 \\
\hline Fluorescent & $20.8-476.7$ & 1.05 & 14 \\
\hline Fluorescent & $0.1-20$ & 0.1 & 48 \\
\hline Piezoelectric & $2.72-27.2$ & 2.12 & 18 \\
\hline Nonisotopic & / & 0.2 & 10 \\
\hline $\begin{array}{l}\text { Surface plasmon } \\
\text { resonance }\end{array}$ & $2-6$ & 0.2 & 15 \\
\hline $\begin{array}{l}\text { Surface plasmon } \\
\text { resonance }\end{array}$ & / & 1 & 49 \\
\hline $\begin{array}{l}\text { Surface plasmon } \\
\text { resonance }\end{array}$ & / & 1.4 & 50 \\
\hline $\begin{array}{l}\text { Surface plasmon } \\
\text { resonance }\end{array}$ & $0.01-100$ & 0.0015 & This work \\
\hline
\end{tabular}

Note: "I" means undetectable or not available.

lossy plasmon wave at the gold-water interface. It means that the gold-water interface is highly sensitive to the molecular interactions. The intensity changes of eight selected cladding modes responding to the thickness of the small molecular modification layer were demonstrated and exhibited the same trends with the experimental results, i.e., the decreased amplitudes of modes from 1 to 4 and the increased ones from 5 to 8 . All modes showed different sensitivities and the most sensitive modes were also identified to be 4 and 5 and their zoomed spectral changes are shown in the inset. Figure 3e shows the responses of different SPR cladding modes to the thickness of small molecular modification layer from 1 to $3 \mathrm{~nm}$. The mode 4 closely located at left shoulder of the SPR envelope was further confirmed with maximum relative intensity response, which accorded well with the above experimental results. The simulated relative intensities of each cladding modes to $3 \mathrm{~nm}$ modification layer (Fig. 3f) also exhibited the same pattern as the SPR envelope, as the experiment revealed.

To summarize, both the above experimental and simulated results identified that the highest sensitivity plasmonic mode of the fabricated gold film-coated TFBG to surface RI was mode 4, which was located at the left shoulder of the SPR envelope.

\section{Performance evaluation}

As revealed above, the SPR envelope will shift in wavelength with the changed surface RI and then modulate the other phase-matched cladding modes.
The wavelength shift of the SPR envelop was also observed under the different bulk solutions used in the analysis. As a consequence, the intensity response of the biosensor arose from both the biomolecule amount attached on the surface and the selected mode. The mode with the highest sensitivities was changing under different bulk solution conditions, hence possible to cover up the response caused by the target, especially at low concentrations. To mitigate the negative impact caused by the fluctuated bulk RI fluctuations of different samples, we calibrated and tested the reflected spectra under different $E_{2}$ concentrations in the DI water. Under the same bulk solution conditions, the surface RI change caused by the EEs can be quantified with the intensity response of TFBG under the selected specific mode. More EEs competed with more $\mathrm{E}_{2}-\mathrm{STV}$ conjugates released from the resin$h E R \alpha$ LBD, which induced their more attachment and hence higher RIs on the TFBG surface. As a consequence, the stronger red shifted SPR envelope caused less attenuation of our selected resonance mode, hence resulting in the stronger relative intensity modulation of narrow cladding mode. All the above provides the theoretical basis for the ultrasensitive quantification of EEs.

Based on the optimized assay conditions (Fig. 3 and Figs. S8 and S9), the performance of TFBG-based SPR biosensor platform was evaluated by using $E_{2}$ as the model analyte for EEs. Although $1 \%(\mathrm{w} / \mathrm{v})$ sodium dodecyl sulfate (SDS) solution in phosphate-buffered saline (PBS) was reported to regenerate the antibody-antigen interaction on the nanocoated fiber surface $^{34}$, the DTB-modified plasmonic optic fiber surface is expected to be stably regenerated by using the washing buffer $(0.5 \%$ SDS, pH 1.9) in the light of the binding between STV and DTB ${ }^{31}$. We first tested the stability of temporal spectrum responses for different concentrations of $E_{2}$ (Fig. 4). The representative realtime intensity curve revealed the stable and targetdependent optical response to $E_{2}$ with the concentration varying from $0.1 \mathrm{ng} \mathrm{ml}^{-1}$ gradually increasing to $10,000 \mathrm{ng} \mathrm{ml}^{-1}$, with a dynamic range of more than five orders of magnitude. The signal was reproducible with attenuation $<0.02 \mathrm{~dB}$ after the plasmonic optic fiber surface was regenerated.

We further evaluated the feasibility of ultrasensitive detection for EEs via the TFBG-based SPR biosensor platform. The spectrum response of monitored SPR mode under different concentrations of $E_{2}$ is recorded in Fig. 5a. It was observed that higher $E_{2}$ concentrations were accompanied with lower optical intensities. To better quantify the targets, we use the optical intensity change ( $\Delta$ Intensity, which is defined as the intensity difference between the base intensity in DI water before reaction and the intensity after certain reactions) to test the sensitivity of this biosensor for EEs. 

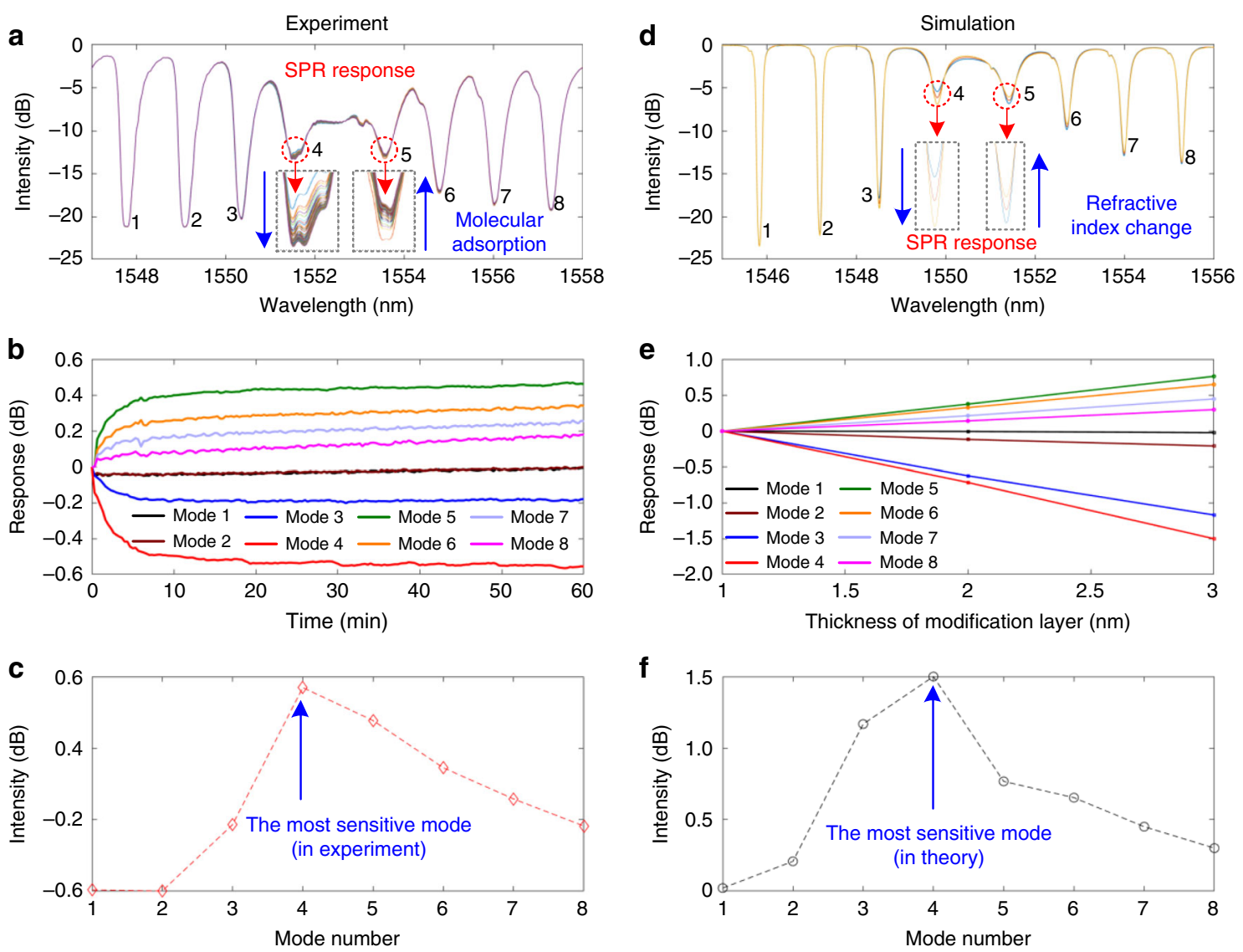

Fig. 3 Optimization of plasmonic sensing characteristics. a Experimental SPR spectrum response of the plasmonic optical fiber when modified with the DTB-PEG-SH on the gold film in $1 \mathrm{~h}$ (inset: zoomed spectral changes of the two most sensitive modes 4 and 5); $\mathbf{b}$ Relative intensity change of the modes 1-8 during the whole DTB-PEG-SH modification; c Total intensity change of modes $1-8$ after $1 \mathrm{~h}$ reaction; $\mathbf{d}-\mathbf{f}$ The simulated results corresponding to the conditions in panels $\mathbf{a}-\mathbf{c}$, in good agreement with the experimental results.

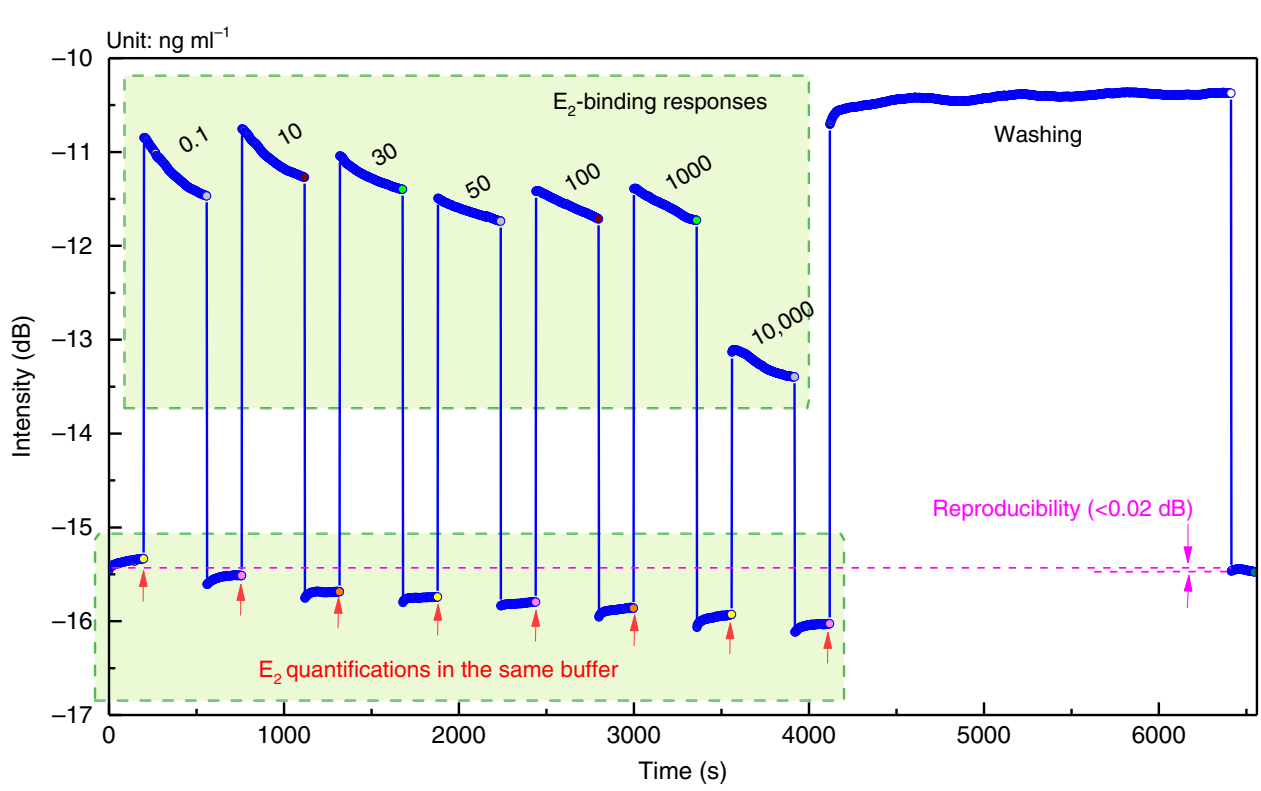

Fig. 4 Stability of gold film-coated TFBG-based SPR biosensor using hERa LBD as a recognition element for EE detection. Representative real-time intensity curve responding to different concentrations of $\mathrm{E}_{2}$ and reproducibility by washing buffer. 

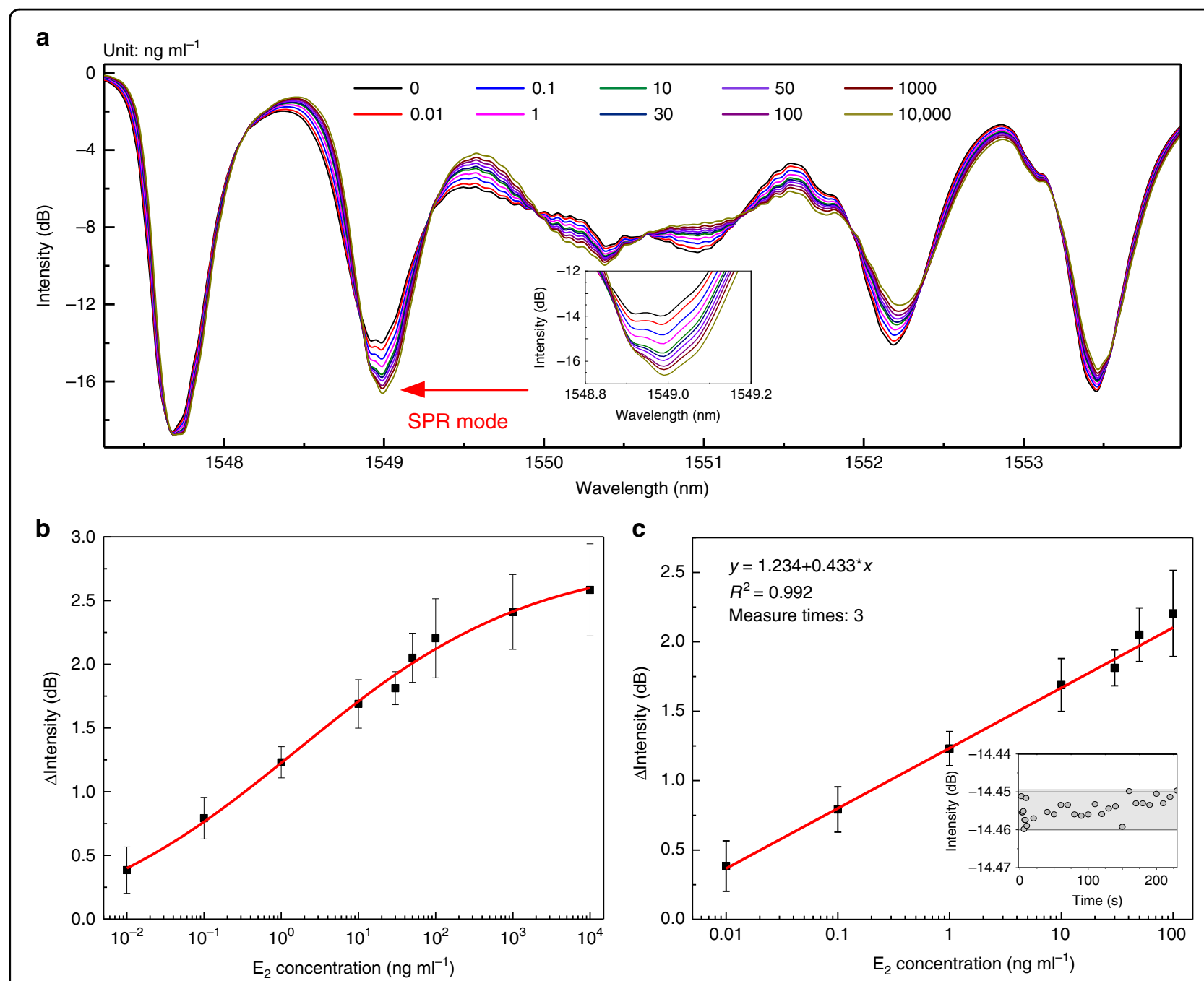

Fig. 5 Sensitivities of gold film-coated TFBG-based SPR biosensor using hERa LBD as a recognition element for EEs detection. a Spectral response of SPR mode versus different $E_{2}$ concentrations; $\mathbf{b}$ variations of $\Delta$ Intensity with the concentration of $E_{2}$ and logarithmic calibration plot; c linear range and corresponding calibration plot to detect $E_{2}$. Inset: intensity fluctuation of a blank sample. The error bars correspond to the standard deviation of the data points in triplicate experiments.

We recorded the $\Delta$ Intensity of both spectra from 1545 to $1556 \mathrm{~nm}$ and mode 4 (inset of Fig. 5a) and related them to the concentrations of $E_{2}$ (Fig. 5b). A logistic functionfitted calibration curve revealed the linear range of $0.01-100 \mathrm{ng} \mathrm{ml}^{-1}$ between the intensity and $\mathrm{E}_{2}$ concentration with a slope of 0.433 and linearity of 0.99 (Fig. 5c, inset: intensity fluctuation of the blank sample). A dramatically linear dynamic range of four orders of magnitude was achieved with a linear fitting function of $y$ $=1.234+0.433^{*} x$. The limit of detection (LOD) of this biosensor toward $E_{2}$, defined as the concentration in which the signal to noise ratio is 3 , was calculated to be $1.5 \times 10^{-3} \mathrm{ng} \mathrm{ml}^{-1}$, considering that the standard deviation of blank was $0.003 \mathrm{~dB}$ (inset of Fig. 5c) ${ }^{35}$. It was one order lower than that of the defined maximal $\mathrm{E}_{2}$ level in drinking water, i.e., $8.0 \times 10^{-2} \mathrm{ng} \mathrm{ml}^{-1}$ set by the Japanese government. The LOD of this biosensor for EE detection was compared with other reported nER-based biosensors (Table 2), and the sensitivity superiority of this biosensor is impressive.

We attributed the sensitivity superiority of this biosensor to the following reasons. Through compensating the red shift of the SPR envelope by using the same bulk solution for testing, the gold-water interface of this biosensor was proven to be highly sensitive to a small change of RIs. Moreover, the EE-induced sensing interface change was characterized by the biological macromolecule of $\mathrm{E}_{2}-\mathrm{STV}$ conjugate through the rational sensing design, which greatly amplified the signals.

The broad sensing capability of this biosensor arises from the specific binding capability between EEs and nERs. To demonstrate its capability for detecting broad 
Table 2 Recovery of $E_{2}$ in real-water samples using the TFBG-based SPR biosensor $(n=3)$.

\begin{tabular}{|c|c|c|c|c|}
\hline Sample & $\begin{array}{l}\text { Spiked } \\
E_{2} \text { (ng } \\
\mathrm{ml}^{-1} \text { ) }\end{array}$ & $\begin{array}{l}\text { Detected } E_{2} \\
\left(\mathrm{ng} \mathrm{ml}^{-1}\right)\end{array}$ & Recovery (\%) & $\begin{array}{l}\text { Coefficient } \\
\text { variation (\%) }\end{array}$ \\
\hline \multirow[t]{3}{*}{ Tap water } & 0 & / & / & / \\
\hline & 1 & $0.91 \pm 0.06$ & 91 & 6 \\
\hline & 10 & $11.23 \pm 0.49$ & 112 & 5 \\
\hline \multirow[t]{3}{*}{ Pond water } & 0 & / & / & / \\
\hline & 1 & $1.06 \pm 0.10$ & 106 & 10 \\
\hline & 10 & $9.80 \pm 0.69$ & 98 & 7 \\
\hline
\end{tabular}

Note: "/" means undetectable or not available.

ranges of EEs, we selected six phthalate esters, which were potential EEs widely reported by numerous stu$\operatorname{dies}^{31,36}$, for investigation. The normalized signal variations responding to $10,000 \mathrm{ng} \mathrm{ml}^{-1}$ phthalate esters, including butyl benzyl phthalate (BBP), diisopentyl phthalate (DIPP), diethyl phthalate (DEP), dimethyl phthalate (DMP), and dioctyl phthalate (DOP), compared with that caused by $10 \mathrm{ng} \mathrm{ml}^{-1} \mathrm{E}_{2}$ are summarized in Fig. S10. The signal decreased in the order BBP > DIPP > DEP > DMP, indicating the relatively weaker estrogen-agonist potencies; however, DOP showed a comparable signal as the control sample, indicating no evidence of estrogenic activities measured by this hER $\alpha$ based biosensor. The results obtained above are consistent with previous studies ${ }^{31,36}$.

Moreover, the interaction of $\mathrm{hER} \alpha \mathrm{LBD}$ with the investigated phthalate esters was simulated by molecular docking and MD. The complex of hER $\alpha$ LBD and four phthalate esters exhibited stable docking poses with the molecular docking scores (Dscores) in the order $\mathrm{BBP}<$ DIPP $<$ DEP $<$ DMP (Fig. S11a). The MD simulations also revealed that the binding energy of EE-hER $\alpha$ LBD complex structures showed the same changing trend as the Dscore (Fig. S11b). However, no stable docking pose or Dscore was generated for the complex of DOP and hER $\alpha$ LBD, indicating that DOP was not suitable for hERa LBD binding. The in silico studies that predicted the estrogenagonist potencies of the phthalate esters are given as follows: $\mathrm{BBP}>\mathrm{DIPP}>\mathrm{DEP}>\mathrm{DMP}$, while the hERa-active potencies of DOP was negligible. The above in silico simulation results accorded well with the experimental results, which further confirmed the biosensing capability of this technique towards broad ranges of EEs.

To demonstrate its capability for practical and realwater samples, two types of real-water samples including laboratory tap water and pond water from the campus of Tsinghua University spiked with different concentrations of $E_{2}$ were detected and evaluated by using the biosensing platform. Only the pond sample was filtered through a
$0.45 \mu \mathrm{m}$ filter (Millipore Corp., Bedford, MA) before spiking. Two spiked concentrations $\left(1 \mathrm{ng} \mathrm{ml}{ }^{-1}, 10 \mathrm{ng}\right.$ $\mathrm{ml}^{-1}$ ) of $\mathrm{E}_{2}$ were chosen according to the sensitivity of our developed biosensor. The concentrations measured by this biosensing platform were compared with spiked concentrations, and results are listed in Table 3. When the non-spiked samples were pumped into the biosensor, no significant change in signal was captured, indicating that EEs were non-detectable in any samples by using this technique. For spiked samples, both for the case of low and high $\mathrm{E}_{2}$ concentrations, the calculated concentrations were close to original spiked values. Notably, the recovery rates in the pond water were more satisfactory than in the laboratory tap samples, considering that the tap water comes from deep groundwater sources and the hardness of this tap water is in the range of $290-350 \mu \mathrm{g} \mathrm{ml}^{-1}$ for calcium carbonate $^{37}$. The high hardness could be one of the possible reasons affecting the interaction between $\mathrm{hER} \alpha$ and EEs, hence the biosensor signal. In summary, the recovery rates of $E_{2}$ ranged from 91 to $112 \%$, demonstrating the satisfactory accuracy of this biosensor and indicating the application potential in real-water samples with a simple pretreatment.

\section{Discussion}

In summary, a label-free plasmonic gold film-coated TFBG-based SPR biosensor using hER $\alpha$ LBD as the biological recognition element was developed to achieve the ultrasensitive detection of EEs. An $E_{2}-S T V$ conjugate consisting of an STV moiety and an $\mathrm{E}_{2}$ moiety was designed to compete with the EEs in the environmental samples to bind with the hER $\alpha$ LBD in the form of resin-hER $\alpha$ LBD complex. Once more EEs existed, more $\mathrm{E}_{2}-\mathrm{STV}$ conjugates were released. The STV moiety of the released conjugates bound with the DTB molecules modified on the TFBG, following with the stronger relative intensity modulation of narrow cladding mode. The "turn-on" sensing mode realized the detection of EEs down to $1.5 \times 10^{-3} \mathrm{ng} \mathrm{ml}^{-1}$ EEQ with a linear range of four orders of magnitude. 
Table 3 Eleven estradiol derivatives used in the simulation.

Number Structure

The proposed in-fiber plasmonic biosensor shows a minimal cross-sensitivity to temperature and its fabrication does not impact the structural integrity of the fiber. The biosensor is able to perform for the in-field continuous detection of estrogenic endocrine disruptors, meeting the highly desired demand for the timely monitoring of environmental status. Meanwhile, integrating such a fiber sensor with a hypodermic needle on the other hand would allow similar measurements, as portable onsite and in-field analysis in health monitoring. To realize a portable instrumentation for in-field measurement, we can use a real-time interrogation scheme by replacing the wavelength interrogation by optical power detection. In this case, a tunable laser (TLS) can be used as a source instead of broadband light source (BBS), together with a photodiode as detector and an analog-to-digital converter to obtain the desired data (to replace the optical spectrum analyzer (OSA)). The function of the TLS is matching the wavelength of the most sensitive SPR mode of the TFBG, so once the sensor is characterized it can be replaced by a common laser (for example, a compact VCSEL). This technique relies on the principle of edge filtering so that the optical power change is produced as a result of the wavelength shift of the mode with respect to the fixed wavelength of the laser source.

Moreover, the platform demonstrated here was able to be extended for detecting a variety of endocrine disruptors by using different nuclear receptors. Endocrine disruptors are chemical compounds that mimics or interferes with the normal actions of hormones in the body via the specific interaction with the nuclear receptors. Bearing the similar biochemical characteristics with
ER, other nuclear receptors, such as androgen receptor, thyroid receptor, and progesterone receptor, are expected to be integrated with the TFBG-based SPR biosensing platform for ultrasensitive detection of other endocrine disruptors.

Last but not least, true multidisciplinary efforts between photonics and bio- and electro-chemistry groups have led to impressive environmentally and clinically ${ }^{38}$, together with emerging renewable energy ${ }^{39-42}$, relevant applications for many substances that require detection and quantification. It is these efforts that are allowing the full potential of such optical fiber sensors to be reached and be able to exploit the extraordinary "Resolution + Specificity + Reproducibility" with fiber gratings and SPR in simple configurations and the combination of other advanced sensing technologies ${ }^{43-45}$.

\section{Materials and methods \\ Materials and reagents}

DTB-PEG-SH-5K was bought from NANOCS. Ni-NTA agarose resin was purchased from GE. SDS, $E_{2}$, isopropyl- $\beta$-D-thiogalactopyranoside, dimethyl sulfoxide, $\beta$-mercaptoethanol, nonidet P400, BSA, SDS, and other chemicals used for buffers and solvents were purchased from Sigma-Aldrich. All buffers were prepared by DI water $(18.2 \mathrm{M} \Omega \mathrm{cm})$.

\section{Gold film-coated TFBG-based SPR biosensor platform}

Figure 6a shows the experimental set-up of gold filmcoated TFBG-based SPR biosensor. The plasmonic TFBG was excited by a BBS (Golight, OS-EB-S-D-1450-400-300 -FA) with the wavelength range of $1250-1650 \mathrm{~nm}$ 

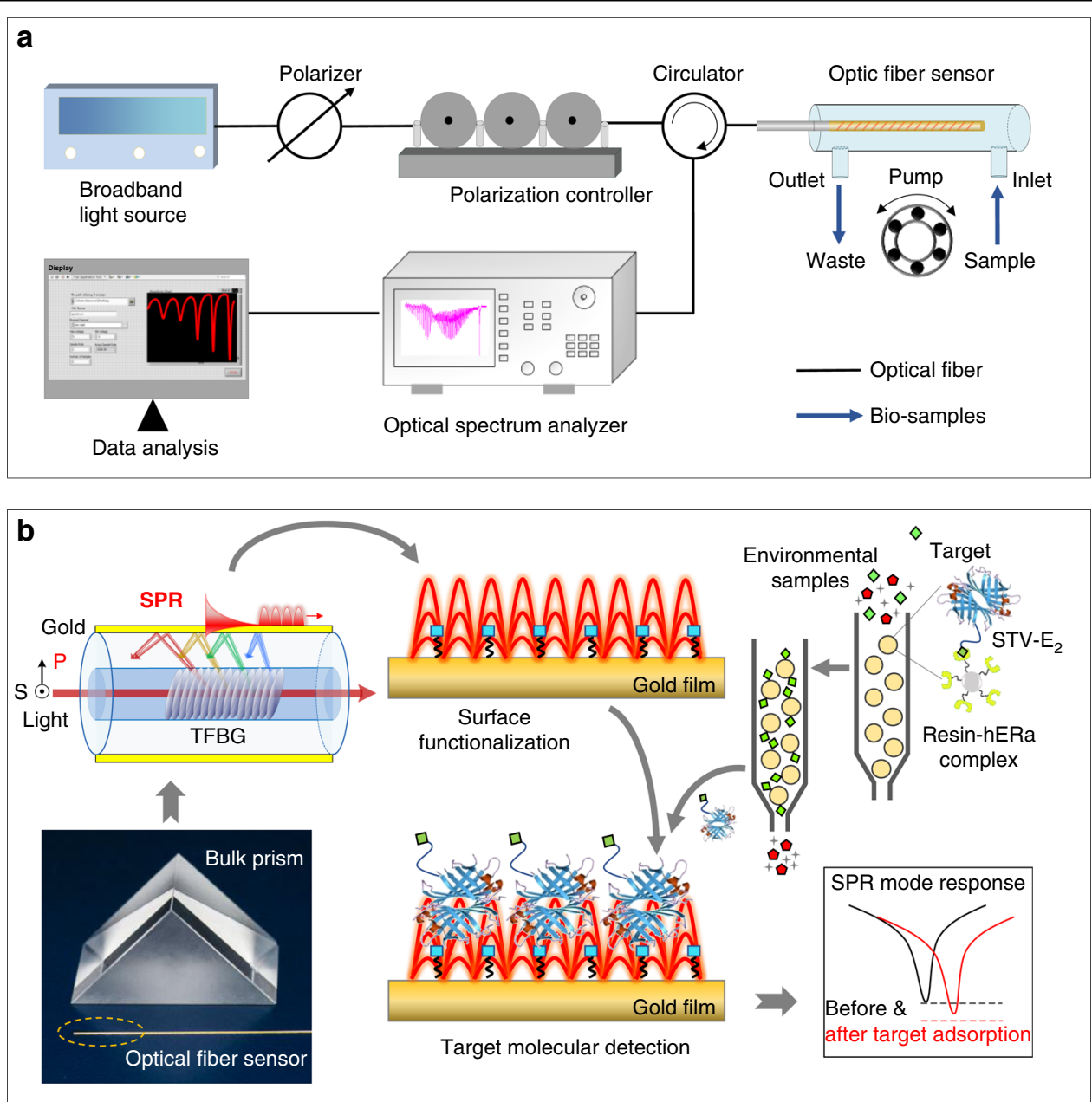

Fig. 6 Plasmonic gold film-coated TFBG-based SPR biosensor using hERa LBD for ultrasensitive EE detection. a Schematic diagram of the biosensor set-up; $\mathbf{b}$ schematic illustration of the sensing mechanism for detection of broad ranges of EEs by using hERa LBD as the biological recognition element. In brief, the gold film-coated fiber surface is tethered with the DTB molecules, and the unbound $E_{2}-S T V$ conjugates are captured via the STV-DTB affinity interaction into the range of SPR in red curve, which will change the spectral of fiber sensors.

(power density of $-10 \mathrm{dBm} \mathrm{nm}^{-1}$ ) to provide superfine plasmonic spectral combs. Its reflection spectrum was monitored by an OSA with a high wavelength resolution of $0.02 \mathrm{~nm}$ (Yokogawa, AQ6370B). The signals were recorded continuously every $20 \mathrm{~s}$ using data analyzer, which was coded in our laboratory using LabVIEW. A linear polarizer and a polarization controller were placed upstream of the circulator to adjust and orient the state of polarization of light launched into the fiber grating so as to provide the strongest SPR excitation. The photograph of self-developed SPR biosensor is shown in Fig. S12.

To fabricate the gold film-coated TFBG, 1-cm-long TFBG was inscribed in the core of boron germanium codoped highly photosensitive single-mode fiber (FIBERCORE PS1250/1500) using a $\sim 1110 \mathrm{~nm}$ period uniform phase mask and a laser emitting at $193 \mathrm{~nm}$ (Bragg Star Industrial, Coherent, Inc.). TFBG with core mode at
$1615 \mathrm{~nm}$ and tens of cladding modes from 1500 to $1600 \mathrm{~nm}$ were achieved. Then an ultra-thin gold film was deposited on the TFBG probe using the sputtering technique (Sky Technology Development, China). To achieve a high-quality coating on the TFBG surface, a layer of chromium with $2-3 \mathrm{~nm}$ thickness was used as a binder in between the silica fiber and the gold film. To ensure a uniform thickness of gold film over the whole fiber surface, the fiber was rotated along its axis during the sputtering. With such design, a robust and uniform gold film with a thickness of $50 \mathrm{~nm}$ was coated over the fiber surface. The gold film thickness was further confirmed by using an ellipsometer (Optical Thickness Meter, Otsuka Electronics). The tilt of the grating is an important parameter that can be used to choose which set of cladding modes is going to be excited. As a result, it makes it possible to adjust the operating range of the sensor in 
order to optimize the response for certain refractive indices. Here the gratings had a tilt of $12^{\circ}$, which maximizes the amplitude of the resonances in aqueous solutions with refractive indices around 1.32-1.34. Finally, the sensor is specially designed with a gold mirror at the end of the fiber. This simple additional process not only offers an increased broadband reflectivity to near $100 \%$ but also makes the light going through the grating twice and doubling the attenuation of each resonance. This configuration also ensures strain-free operation of the sensor to eliminate the effect of cross-sensitivity to strain of the higher-order cladding and plasmonic modes when the sensor is closely attached.

\section{Sensing mechanism}

Figure $6 \mathrm{~b}$ depicts the schematic illustration of the TFBG-based SPR biosensor for the detection of broad ranges of EEs. It is worth mentioning that, as a classic subtype of $n E R s, n E R \alpha$ protein shows estrogen-dependent gene transcriptional activation via their key activation domain-LBD ${ }^{1,5,7}$. Structurally, $\mathrm{nER} \alpha \mathrm{LBD}$ is very conserved and is responsible for the ligand binding via identification of the structural commonalities among diverse estrogenic endocrine disruptors ${ }^{5,46}$. To realize the recognition of broad ranges of EEs, the LBD of human $\mathrm{nER} \alpha$ (hER $\alpha$ LBD) (aa302-552 with three mutations of aa381, 417, 530 from Cys to Ser) encoding plasmid (pET 28a) was self-constructed in our laboratory and expressed in Escherichia coli (BL21 DE3). By adding six His tag to its $\mathrm{N}$-terminal, it was purified by the affinity chromatography via binding to the $\mathrm{Ni}^{2+}$-charged agarose (Ni-NTA His.Bind ${ }^{\circledR}$ resin) with an average diameter of $90 \mu \mathrm{m}$. The purified $31 \mathrm{kDa}$ hER $\alpha$ LBD protein was confirmed by SDS-polyacrylamide gel electrophoresis as shown in Fig. S13. More details are described in our previous studies $^{14}$. The obtained complex of resin-hER $\alpha$ LBD was used as the biological recognition element to avoid the biological activity loss of the eluted hER $\alpha$ LBD protein. An $\mathrm{E}_{2}-\mathrm{STV}$ conjugate consisting of a STV protein moiety and an $E_{2}$ small-molecule moiety was designed to compete with the EEs in the environmental samples to bind with the hER $\alpha$ LBD in the form of bound state (resin-hER $\alpha$ LBD complex). Once more EEs existed, more $\mathrm{E}_{2}-\mathrm{STV}$ conjugates were released. After a simple centrifugal separation, the supernatant with unbound conjugates was fed into the flow cell of the TFBG-based SPR biosensor. The STV moiety of conjugates bound with the DTB molecules modified on the optical fiber via the STV-DTB affinity interaction ${ }^{47}$, which was followed with an increase in the intensity of cladding mode with SPR excitation. Normally, the more the EEs, the more red shift the SPR envelope, resulting in the stronger relative intensity modulation of narrow cladding mode. It demonstrated a typical "turn-on" sensing mode.

\section{Design of $E_{2}-S T V$ conjugate}

To realize the conjugate-mediated measurement for the quantification of EEs in environmental samples, the $\mathrm{E}_{2}-\mathrm{STV}$ conjugate that was able to interact with the $\mathrm{hER} \alpha$ LBD efficiently was rationally designed. In order to avoid the steric hindrance caused by STV, various link arms were introduced to the $\mathrm{E}_{2}-\mathrm{STV}$ conjugates, containing five estradiol derivatives with flexible joints named 4,8 , 11,16 , and 20 and six estradiol derivatives with rigid joints named r3, r5, r8, r11, r16, and r20 as shown in Table 3. The interaction of estradiol derivatives and hER $\alpha$ LBD were simulated by MD simulation, respectively. The crystal structure of $\mathrm{E}_{2}$-hER $\alpha$ LBD (PDB ID: 1GWR) obtained from the protein database RCSB PDB (http:// www.rcsb.org) was first checked in Autodock Tools (http://autodock.scripps.edu/resources/adt) to remove unnecessary water molecules and add lost hydrogen atoms. Next, 11 kinds of estradiol derivatives were aligned to the original $E_{2}-h E R \alpha$ LBD crystal structure. Then the complexes of estradiol derivatives and hER $\alpha$ LBD were used as the initial conformation for MD simulation, which was carried out in Gromacs 2016.4 under the GROMOS96 43a1 force field. In the MD simulations, the complex was first solvated with simple point charge water molecules in a dodecahedral box and the minimum distance between complexes and the solvent box was $1.5 \mathrm{~nm}$. The $\mathrm{Na}^{+}$and $\mathrm{Cl}^{-}$ions were then added to the solvent box for charge balance. All MD processes underwent energy minimization, temperature coupling $(300 \mathrm{k})$, pressure coupling ( $1 \mathrm{bar})$, and $10 \mathrm{~ns}$ simulation. The estradiol derivative with the most stable binding capability to hER $\alpha$ LBD was chosen for the synthesis of the conjugate by Shanghai Qianyan Biotechnology Co., Ltd. (China). The nuclear magnetic resonance identification report of the synthesized estradiol derivative 8 is demonstrated in Fig. S14.

\section{Surface functionalization of plasmonic optical fiber sensor}

To realize the signal record of binding the conjugate on the surface of TFBG, the plasmonic optical fiber with gold film on the surface was further modified with the DTB molecule, which showed specific binding with the STV moiety of $\mathrm{E}_{2}-\mathrm{STV}$ conjugate. Notably, the moderate binding affinity between STV and DTB enabled the regeneration of the fiber surface for reuse with less loss of activity, thereby allowing semi-continuous automated water monitoring. We used a well-established strategy to immobilize functional groups/molecules onto thin SPR films by synthesizing thiol-end DTB derivative (DTBPEG-SH), forming self-assembled monolayers on gold film surfaces via Au-S bond (Fig. 1a). Briefly speaking, the gold-coated fiber surface was first treated by oxygen plasma for $5 \mathrm{~min}$ to remove possible contaminants and then thoroughly rinsed with DI water. The clean fiber was 
subsequently treated with $2 \mathrm{ml}$ DTB solution $(40 \mu \mathrm{M}$ DTB-PEG-SH and $200 \mu \mathrm{M} \mathrm{H}_{3} \mathrm{PO}_{4}$ ) at a flow rate of approximately $1 \mathrm{ml} \mathrm{min}^{-1}$ for $3 \mathrm{~h}$ at room temperature to form the binding layer and then thoroughly rinsed with $10 \mathrm{mM}$ PBS, pH 7.4. Finally, it was immersed in the BSA solution ( $5 \mathrm{mg} \mathrm{ml}^{-1}$ in $10 \mathrm{mM}$ PBS buffer, $\left.\mathrm{pH} 7.4\right)$ for $1 \mathrm{~h}$ to block its non-specific binding sites. The surfacefunctionalized gold-coated TFBG fiber was stored at $4{ }^{\circ} \mathrm{C}$ before use.

\section{Quantification of EEs}

After the optimization of procedures for EEs detection, $500 \mu \mathrm{l}$ of $5 \mu \mathrm{g} \mathrm{ml}^{-1} \mathrm{E}_{2}-\mathrm{STV}$ conjugate was first added into 58 pmol resin-hER $\alpha$ LBD, and the mixture was maintained at $4{ }^{\circ} \mathrm{C}$ overnight for the affinity binding of $\mathrm{E}_{2}$ with $h E R \alpha$ LBD. Next, the mixture was centrifuged at $3000 \mathrm{rpm}$ for $2 \mathrm{~min}$ and $450 \mathrm{ml}$ of supernatant was discarded. Subsequently, $500 \mu \mathrm{l}$ of $\mathrm{E}_{2}$ standard solutions or test samples were added and rotationally mixed at $4{ }^{\circ} \mathrm{C}$ for $8 \mathrm{~h}$ to compete for the $\mathrm{E}_{2}$-binding motif with hER $\alpha \mathrm{LBD}$. The dissociated conjugate was then introduced to bind the DTB on the TFBG fiber surface for 6 min, which was detected by the SPR excitation after stabilizing the probe through the DI water. After the signal detection, the $\mathrm{E}_{2}-\mathrm{STV}$ conjugate was able to be eluted using $5 \mathrm{ml}$ of $0.5 \%$ SDS ( $\mathrm{pH} 1.9$ ) for $25 \mathrm{~min}$ to ensure the regeneration of sensing surface. The whole detection procedure took place within $40 \mathrm{~min}$.

The amount of EEs in test samples was represented by the estrogenic activities, which was characterized by the EEQ by using $E_{2}$ as the reference compound.

\section{Acknowledgements}

This research was supported by National Key Research and Development Project (2019YFD1100505), the Key Program of National Natural Science Foundation of China (62035006), and the National Natural Science Foundation of China $(21976100,61975068,62005101,62011530459)$. The authors are grateful to Professor Jacques Albert of Carleton University (Canada) for valuable discussions and the support of TFBG-SPR simulations.

\section{Author contributions}

T.G. and X. Zhou conceived the sensors, supervised the project, and analyzed the data. L.L., X. Zhang, and Q.Z. fabricated the sensors and the materials and carried out experiments. Theoretical analyses were done by X. Zhang, Q.Z., X. Zhou, and T.G. All authors contributed to the preparation of the manuscript.

\section{Data availability}

All data needed to evaluate the conclusions in the paper are present in the paper and/or the Supplementary Materials. Data from these experiments and MATLAB code used for data analyses are available from the corresponding authors upon reasonable request.

\section{Conflict of interest}

The authors declare no competing interests.

Supplementary information The online version contains supplementary material available at https://doi.org/10.1038/s41377-021-00618-2.
Received: 29 May 2021 Revised: 14 August 2021 Accepted: 17 August 2021 Published online: 07 September 2021

\section{References}

1. Hunt, J. P. et al. Rapid, portable detection of endocrine disrupting chemicals through ligand-nuclear hormone receptor interactions. Analyst 142, 4595-4600 (2017).

2. Ismail, N. A. H. et al. Quantification of multi-classes of endocrine-disrupting compounds in estuarine water. Environ. Pollut. 249, 1019-1028 (2019).

3. Yao, B. et al. Occurrence and estrogenic activity of steroid hormones in Chinese streams: a nationwide study based on a combination of chemical and biological tools. Environ. Int. 118, 1-8 (2018).

4. Memon, A. G. et al. Ultrasensitive colorimetric aptasensor for $\mathrm{Hg}^{2+}$ detection using Exo-III assisted target recycling amplification and unmodified AuNPs as indicators. J. Hazard. Mater. 384, 120948 (2020).

5. Brzozowski, A. M. et al. Molecular basis of agonism and antagonism in the oestrogen receptor. Nature 389, 753-758 (1997).

6. Rodriguez-Mozaz, S. et al. Biosensors for environmental monitoring of endocrine disruptors: a review article. Anal. Bioanal. Chem. 378, 588-598 (2004).

7. Björnström, L. \& Sjöberg, M. Mechanisms of estrogen receptor signaling: convergence of genomic and nongenomic actions on target genes. Mol. Endocrinol. 19, 833-842 (2005).

8. Carvalho, R. N. et al. Mixtures of chemical pollutants at European legislation safety concentrations: how safe are they? Toxicol. Sci. 141, 218-233 (2014).

9. Seifert, M., Haindl, S. \& Hock, B. Development of an enzyme linked receptor assay (ELRA) for estrogens and xenoestrogens. Anal. Chim. Acta 386, 191-199 (1999).

10. Garrett, S. D., Lee, H. A. \& Morgan, M. R. A. A nonisotopic estrogen receptor-based assay to detect estrogenic compounds. Nat. Biotechnol. 17, 1219-1222 (1999).

11. Shelby, M. D. et al. Assessing environmental chemicals for estrogenicity using a combination of in vitro and in vivo assays. Environ. Health Perspect. 104, 1296-1300 (1996).

12. Kuiper, G. G. J. M. et al. Comparison of the ligand binding specificity and transcript tissue distribution of estrogen receptors a and $\beta$. Endocrinology $\mathbf{1 3 8}$, 863-870 (1997)

13. Liu, L. H. et al. Facile screening of potential xenoestrogens by an estrogen receptor-based reusable optical biosensor. Biosens. Bioelectron. 97, 16-20 (2017).

14. Liu, L. H. et al. Triple functional small-molecule-protein conjugate mediated optical biosensor for quantification of estrogenic activities in water samples. Environ. Int. 132, 105091 (2019)

15. La Spina, R. et al. Label-free biosensor detection of endocrine disrupting compounds using engineered estrogen receptors. Biosensors 8, 1 (2018).

16. Fechner, P. et al. An advanced biosensor for the prediction of estrogenic effects of endocrine-disrupting chemicals on the estrogen receptor alpha. Anal. Bioanal. Chem. 393, 1579-1585 (2009).

17. Gao, Y., Li, X. X. \& Guo, L. H. Assessment of estrogenic activity of perfluoroalkyl acids based on ligand-induced conformation state of human estrogen receptor. Environ. Sci. Technol. 47, 634-641 (2013).

18. Mo, Z. H., Long, X. H. \& Fu, W. L. A new sandwich-type assay of estrogen using piezoelectric biosensor immobilized with estrogen response element. Anal. Commun. 36, 281-283 (1999).

19. Murata, M. et al. Novel biosensor for the rapid measurement of estrogen based on a ligand-receptor interaction. Anal. Sci. 17, 387-390 (2001).

20. Xia, W. et al. Electrochemical biosensor for estrogenic substance using lipid bilayers modified by Au nanoparticles. Biosens. Bioelectron. 25, 2253-2258 (2010).

21. Asano, H., Maeda, T. \& Shiraishi, Y. Sensitive determination of hexavalent chromium using a microfluidic paper-based analytical device with solid phase extraction. Bunseki Kagaku 70, 379-383 (2021).

22. Caucheteur, C., Guo, T. \& Albert, J. Review of plasmonic fiber optic biochemical sensors: improving the limit of detection. Anal. Bioanal. Chem. 407, 3883-3897 (2015).

23. Allsop, T. D. P. et al. An ultra-sensitive aptasensor on optical fibre for the direct detection of bisphenol A. Biosens. Bioelectron. 135, 102-110 (2019).

24. Lamarca, R. S. et al. Label-free ultrasensitive and environment-friendly immunosensor based on a silica optical fiber for the determination of ciprofloxacin in wastewater samples. Anal. Chem. 92, 14415-14422 (2020). 
25. Pollet, J. et al. Fiber optic SPR biosensing of DNA hybridization and DNA-protein interactions. Biosens. Bioelectron. 25, 864-869 (2009).

26. Albert, J. et al. Tilted fiber Bragg grating sensors. Laser Photonics Rev. 7, 83-108 (2013).

27. Guo, T. Fiber grating-assisted surface Plasmon resonance for biochemical and electrochemical sensing. J. Lightwave Technol. 35, 3323-3333 (2017)

28. Caucheteur, C. et al. Ultrasensitive plasmonic sensing in air using optical fibre spectral combs. Nat. Commun. 7, 13371 (2016).

29. Cai, S. S. et al. Narrow bandwidth fiber-optic spectral combs for renewable hydrogen detection. Sci. China Inf. Sci. 63, 222401 (2020).

30. Chen, Y. Y. et al. Optical biosensors based on refractometric sensing schemes: a review. Biosens. Bioelectron. 144, 111693 (2019).

31. Zhu, Q. et al. In silico study of molecular mechanisms of action: estrogenic disruptors among phthalate esters. Environ. Pollut. 255, 113193 (2019).

32. Erdogan, T. \& Sipe, J. E. Tilted fiber phase gratings. J. Optical Soc. Am. A 13, 296-313 (1996).

33. $\mathrm{Fu}, \mathrm{L}$. et al. Discrimination of bulk and surface refractive index change in plasmonic sensors with narrow bandwidth resonance combs. ACS Sens. 6, 3013-3023 (2021). https://doi.org/10.1021/acssensors.1c00906.

34. Chiavaioli, F. et al. Femtomolar detection by nanocoated fiber label-free biosensors. ACS Sens. 3, 936-943 (2018).

35. Chiavaioli, F. et al. Towards a uniform metrological assessment of gratingbased optical fiber sensors: from refractometers to biosensors. Biosensors 7, 23 (2017).

36. Takeuchi, S. et al. Differential effects of phthalate esters on transcriptional activities via human estrogen receptors $\alpha$ and $\beta$, and androgen receptor. Toxicology 210, 223-233 (2005).

37. Zhou, X. H. et al. A reusable evanescent wave immunosensor for highly sensitive detection of bisphenol A in water samples. Sci. Rep. 4, 4572 (2014).

38. Guo, T. et al. Plasmonic optical fiber-grating immunosensing: a review. Sensors 17, 2732 (2017)
39. Yuan, Y. et al. Electrochemical surface Plasmon resonance fiber-optic sensor: in situ detection of electroactive biofilms. Anal. Chem. 88 7609-7616 (2016).

40. Lao, J. J. et al. In situ plasmonic optical fiber detection of the state of charge of supercapacitors for renewable energy storage. Light. Sci. Appl. 7, 34 (2018).

41. Si, Y. et al. Electrochemical plasmonic fiber-optic sensors for ultra-sensitive heavy metal detection. J. Lightwave Technol. 37, 3495-3502 (2019).

42. Zhang, X. J. et al. In situ determination of the complex permittivity of ultrathin $\mathrm{H}_{2}$-infused palladium coatings for plasmonic fiber optic sensors in the near infrared. J. Mater. Chem. C 6, 5161-5170 (2018).

43. Shi, Y. Z. et al. Chirality-assisted lateral momentum transfer for bidirectional enantioselective separation. Light. Sci. Appl. 9, 62 (2020).

44. Toropov, N. et al. Review of biosensing with whispering-gallery mode lasers. Light. Sci. Appl. 10, 42 (2021)

45. Pan, T. et al. Biophotonic probes for bio-detection and imaging. Light. Sci. Appl. 10, 124 (2021).

46. Fang, $\mathrm{H}$. et al. Structure-activity relationships for a large diverse set of natural, synthetic, and environmental estrogens. Chem. Res. Toxicol. 14, 280-294 (2001).

47. Wang, R. Y. et al. T-T mismatch-driven biosensor using triple functional DNAprotein conjugates for facile detection of $\mathrm{Hg}^{2+}$. Biosens. Bioelectron. 78, 418-422 (2016).

48. Wang, D. et al. A recombinant estrogen receptor fragment-based homogeneous fluorescent assay for rapid detection of estrogens. Biosens. Bioelectron. 55, 391-395 (2014).

49. Hock, B., Seifert, M. \& Kramer, K. Engineering receptors and antibodies for biosensors. Biosens. Bioelectron. 17, 239-249 (2002).

50. Habauzit, D. et al. Determination of estrogen presence in water by SPR using estrogen receptor dimerization. Anal. Bioanal. Chem. 390, 873-883 (2008). 